\title{
Reconfigurable Intelligent Surfaces Assisted Communications with Discrete Phase Shifts: How Many Quantization Levels are Required to Achieve Full Diversity?
}

\author{
Peng Xu, Member, IEEE, Gaojie Chen, Senior Member, IEEE, Zheng Yang, Member, IEEE, \\ and Marco Di Renzo, Fellow, IEEE
}

\begin{abstract}
Due to hardware limitations, the phase shifts of the reflecting elements of reconfigurable intelligent surfaces (RISs) need to be quantized into discrete values. This letter aims to unveil the minimum required number of phase quantization levels $L$ in order to achieve the full diversity order in RISassisted wireless communication systems. With the aid of an upper bound of the outage probability, we first prove that the full diversity order is achievable provided that $L$ is not less than three. If $L=2$, on the other hand, we prove that the achievable diversity order cannot exceed $(N+1) / 2$, where $N$ is the number of reflecting elements. This is obtained with the aid of a lower bound of the outage probability. Therefore, we prove that the minimum required value of $L$ to achieve the full diversity order is $L=3$. Simulation results verify the theoretical analysis and the impact of phase quantization levels on RISassisted communication systems.
\end{abstract}

Index Terms-Reconfigurable intelligent surface, discrete phase shifts, phase errors, outage probability, diversity order.

\section{INTRODUCTION}

Reconfigurable intelligent surfaces (RISs) have recently received significant attention due to their ability to intelligently reconfigure the wireless environment by integrating passive reflectors on flat surfaces [1]-[3]. Precisely, each element of the RIS can control the amplitude and phase of the reflected signal, such that multiple reflected signals can be co-phased through passive beamforming. Compared with other technologies, such as relaying, RISs do not need to use radio-frequency chains and enjoys a convenient and low-cost deployment [4], [5].

Due to their potential benefits in the context of wireless communications, RISs have recently been employed for several applications, which include beamforming [6], [7], nonorthogonal multiple access [8], [9], physical layer security [10], relaying [5], [11], etc. These existing works have demonstrated that a suitable design of the phase shifts of the reflecting elements is necessary to reap the advantages of RIS-aided transmission. However, most of these existing works assume that the phase shifts of the reflectors are continuous variables, which is difficult to implement in practice due to hardware limitations. Motivated by these considerations, recent works

$\mathrm{P} . \mathrm{Xu}$ is with Chongqing Key Laboratory of Mobile Communications Technology, School of Communication and Information Engineering, Chongqing University of Posts and Telecommunications, Chongqing, 400065, China.

G. Chen is with School of Engineering, University of Leicester, Leicester LE1 7RH, U.K. (gaojie.chen@leicester.ac.uk).

Z. Yang is with Fujian Provincial Engineering Technology Research Center of Photoelectric Sensing Application, Key Laboratory of OptoElectronic Science and Technology for Medicine of Ministry of Education, Fujian Normal University, Fuzhou 350007, China (e-mail: zyfjnu@163.com).

M. Di Renzo is with Université Paris-Saclay, CNRS and CentraleSupélec, Laboratoire des Signaux et Systèmes, Gif-sur-Yvette, France. (email: marco.direnzo@centralesupelec.fr). have investigated the performance of RIS-assisted systems in the presence of quantization phase errors (e.g., [7], [9], [12][15]). The works in [7] and [9] solved optimization problems with respect to the discrete phase shifts. The works in [12][15] analyzed the average signal-to-noise-ratio (SNR) and the achievable data rate. The works in [12]-[15] demonstrated that the average SNR scales with $N^{2}$ for large values of $N$, where $N$ denotes the number of reflecting elements. However, the exact relation between the diversity order and the number of phase quantization levels $L$ for arbitrary $N \geq 2$ was not revealed. Motivated by these considerations, we investigate the diversity order of RIS-assisted transmission in the presence of quantization phase errors for any $N \geq 2$.

More specifically, the main contribution of this letter consists of unveiling the minimum number of required phase quantization levels to achieve the full diversity order $N$. To obtain this goal, the full diversity order of $N$ is first proved to be achievable if $L \geq 3$, by using mathematical induction based on an upper bound of the outage probability. Then, the achievable diversity order is proved not to exceed $\frac{N+1}{2}$ if $L=2$, with the aid of a lower bound of the outage probability conditioned on the event that each phase quantization error is close to the quantization boundary. Numerical results also demonstrate that the loss of the outage performance is negligible if $L \geq 3$, which provides important insights for the design of phase quantization in RIS-assisted systems.

\section{System Model And Preliminaries}

We consider an RIS-aided transmission system, which consists of a single-antenna source $(S)$, a single-antenna destination $(D)$ and an RIS with $N$ reflecting elements. The direct link between $S$ and $D$ is assumed to be weak and it is not considered 1 . The channel coefficients from $S$ to the RIS and from the RIS to $D$ are denoted by the column-vectors $\mathbf{h}_{S I}$ and $\mathbf{h}_{I D}$, respectively. The $n$th elements of $\mathbf{h}_{S I}$ and $\mathbf{h}_{I D}$ are $\left[\mathbf{h}_{S I}\right]_{n} \sim \mathcal{C N}\left(0, \Omega_{S}\right)$ and $\left[\mathbf{h}_{I D}\right]_{n} \sim \mathcal{C N}\left(0, \Omega_{I}\right)$, respectively, $\forall n \in\{1, \ldots, N\}$, where $\mathcal{C N}$ represents the complex Gaussian distributions and $\Omega_{S}$ and $\Omega_{I}$ are the variances of $\left[\mathbf{h}_{S I}\right]_{n}$ and $\left[\mathbf{h}_{I D}\right]_{n}$, respectively. All channel coefficients are assumed to be mutually independent. For each transmission, the received signal at $D$ can be written as follows

$$
y_{D}=\sqrt{P} \eta \mathbf{h}_{S I}^{T} \boldsymbol{\Phi} \mathbf{h}_{I D} x_{S}+w_{D},
$$

where $x_{S}$ is the transmitted signal from $S, \mathbb{E}\left(\left|x_{S}\right|^{2}\right)=1, P$ is the transmit power, $w_{D} \sim \mathcal{C N}\left(0, \delta^{2}\right)$ is the additive white Gaussian noise at $D, \boldsymbol{\Phi}=\operatorname{diag}\left(e^{j \phi_{1}}, e^{j \phi_{2}}, \ldots, e^{j \phi_{N}}\right), \eta \in$

\footnotetext{
${ }^{1}$ We consider the direct link between $S$ and $D$ in Section $\mathbf{V}$
} 
$(0,1]$ is the amplitude reflection coefficient $t^{2}$ and $\phi_{n} \in[0,2 \pi]$ is the phase shift introduced by the $n$th reflecting element.

The optimal value of $\phi_{n}$ is $\phi_{n}^{*}=-\arg \left(\left[\mathbf{h}_{S I}\right]_{n}\left[\mathbf{h}_{I D}\right]_{n}\right), n \in$ $\{1, \ldots, N\}$. Without considering the impact of phase errors, the full diversity order of $N$ can be achieved with this optimal setting [8]. However, only a finite number of quantized values can be considered in practice. Therefore, we consider a finite number of quantization levels $L \geq 2$, where $L$ is a positive integer. Accordingly, the number of quantization bits is $\log _{2} L$. The phase shift range is uniformly quantized into $L$ levels, i.e., $\mathcal{F} \triangleq\left\{0, \frac{2 \pi}{L}, \ldots, \frac{(L-1) 2 \pi}{L}\right\}$, and the phase shift of each reflecting element is designed by mapping its optimal value to the nearest point in $\mathcal{F}$, i.e., $\phi_{n}=\hat{\phi}_{l_{l}}$, where $\left|\phi_{n}^{*}-\hat{\phi}_{l_{l}}\right| \leq \mid \phi_{n}^{*}-$ $\hat{\phi}_{l_{u}} \mid, \hat{\phi}_{l_{l}}, \hat{\phi}_{l_{u}} \in \mathcal{F}$. Then, the phase error $\Theta_{n}=\hat{\phi}_{l_{l}}-\phi_{n}^{*}$ for the $n$th reflecting element is a uniformly distributed random variable in $\left[-\frac{\pi}{L}, \frac{\pi}{L}\right][12]$. Thus, the received SNR at $D$ can be written as follows

$$
\gamma_{D}=\rho \eta^{2}\left|\sum_{n=1}^{N}\right|\left[\mathbf{h}_{S I}\right]_{n}\left[\mathbf{h}_{I D}\right]_{n}\left|e^{j \Theta_{n}}\right|^{2}=\rho \eta^{2} \Omega_{S} \Omega_{I}\left|\sum_{n=1}^{N} g_{n}\right|^{2}
$$

where $\rho \triangleq \frac{P}{\delta^{2}}$ denotes the transmit SNR and $g_{n} \triangleq$ $\frac{\left|\left[\mathbf{h}_{S I}\right]_{n}\left[\mathbf{h}_{I D}\right]_{n}\right| e^{j \Theta_{n}}}{\sqrt{\Omega_{S} \Omega_{I}}}$ is the normalized channel coefficient. From [8], the cumulative distribution function (CDF) of $\left|g_{n}\right|^{2}$ can be expressed as

$$
F_{\left|g_{n}\right|^{2}}(x)=1-2 \sqrt{x} K_{1}(2 \sqrt{x}) \approx-x \ln x, \text { as } x \approx 0,
$$

where $K_{1}(\cdot)$ denotes the modified Bessel function of the second kind.

The outage probability between $S$ and $D$ can be expressed as follows

$$
\begin{aligned}
P_{N}^{\text {out }}(\rho) & =\operatorname{Pr}\left\{\log _{2}\left(1+\rho \eta^{2} \Omega_{S} \Omega_{I}\left|G_{N}\right|^{2}\right)<R_{0}\right\} \\
& =\operatorname{Pr}\left\{\left|G_{N}\right|^{2}<\epsilon_{0} \rho^{-1}\right\},
\end{aligned}
$$

where $G_{N} \triangleq \sum_{n=1}^{N} g_{n}$ and $R_{0}$ denotes the target data rate in bits per channel use (bpcu) and $\epsilon_{0} \triangleq \frac{2^{R_{0}}-1}{\eta^{2} \Omega_{S} \Omega_{I}}$. The diversity order is defined, as a function of $L$, as follows:

$$
d_{N}(L)=\lim _{\rho \rightarrow \infty} \frac{-\log P_{N}^{\text {out }}(\rho)}{\log \rho} .
$$

Remark 1: Although the central limit theorem (CLT) is widely adopted to analyze the averaged SNR of RIS-assisted systems for large values of $N$, it is not suitable to analyze the diversity order. In particular, the CLT-based approximation has an error floor, i.e., the outage probability becomes a constant when $\rho \rightarrow \infty$ as shown in [8].

Remark 2: The CDF of $G_{N}$ is difficult to obtain, since its real part and imaginary part are correlated. Therefore, an exact analytical expression for the outage probability $P_{N}^{\text {out }}$ cannot be obtained, in general. Motivated by these considerations, in the following sections, we derive lower and upper bounds for $P_{N}^{\text {out }}$ in order to study the achievable diversity order for different values of $L$.

2 For simplicity, this letter assumes that all elements have the same amplitude reflection coefficient.

\section{DIVERSITY ORDER FOR $L=3$}

In this section, we introduce an upper bound for the outage probability in (4) and prove that the maximum diversity order $N$ can be achieved in this case. When $L=3$, we have $\Theta_{n} \in\left[-\frac{\pi}{3}, \frac{\pi}{3}\right], \forall n \in\{1, \ldots, N\}$. Before proceeding with the analytical derivation, we introduce two lemmas.

Lemma 1: For $\forall C_{1}, C_{2} \in \mathbb{C}(\mathbb{C}$ denotes the complex domain), $\arg \left(C_{1}+C_{2}\right) \in\left[\varphi_{1}, \varphi_{2}\right]$ holds if $\arg \left(C_{1}\right), \arg \left(C_{2}\right) \in$ $\left[\varphi_{1}, \varphi_{2}\right]$ and $-\frac{\pi}{2} \leq \varphi_{1} \leq \varphi_{2} \leq \frac{\pi}{2}$.

Proof 1: This lemma can be easily obtained based on geometrical relationships among $\tilde{g}_{i}, \tilde{g}_{j}$ and $\tilde{g}_{i}+\tilde{g}_{j}$ in a complex plane, whose details are omitted for brevity.

Lemma 2: For $\forall b, c \in \mathbb{R}^{+}\left(\mathbb{R}^{+}\right.$denotes the positive real domain), and $\forall n \in\{1, \ldots, N\}$, we have 3

(a) $\operatorname{Pr}\left\{\left(\left|g_{n}\right|-a\right)^{2}<b \rho^{-1}\right\} \dot{\leq} \rho^{-1}$, if $0<a<c \rho^{-\frac{1}{2}}$;

(b) $\operatorname{Pr}\left\{\left(\left|g_{n}\right|-a\right)^{2}<b \rho^{-1}\right\} \doteq \rho^{-\frac{1}{2}}$, if $a>0$ being independent of $\rho$, i.e., $a \doteq \rho^{0}$.

where ".”" denotes exponential equality [16], i.e., $f(\rho) \doteq \rho^{d}$ is defined as $\lim _{\rho \rightarrow \infty} \frac{\log f(\rho)}{\log \rho}=d$, and $d \in \mathbb{R}$ is the exponential order of $f(\rho)$. Similarly, $f(\rho) \dot{\leq} \rho^{d}\left(\dot{\geq} \rho^{d}\right)$ is defined as $\lim _{\rho \rightarrow \infty} \frac{\log f(\rho)}{\log \rho} \leq d(\geq d)$.

Proof 2: See Appendix A

In the following two subsections, we derive the outage probability for $N=2$ and $N \geq 2$, respectively.

A. $N=2$

When $N=2, G_{2}=g_{1}+g_{2}$. Since $\left|\Theta_{1}-\Theta_{2}\right| \leq \frac{2 \pi}{3}$ and $G_{2}$ decreases with $\left|\Theta_{1}-\Theta_{2}\right|,\left|G_{2}\right|^{2}$ can be lower bounded

$$
\begin{aligned}
\left|G_{2}\right|^{2} & =\left|g_{2}\right|^{2}+\left|g_{1}\right|^{2}+2\left|g_{2}\right|\left|g_{1}\right| \cos \left(\left|\Theta_{2}-\Theta_{1}\right|\right) \\
& \geq\left|g_{2}\right|^{2}+\left|g_{1}\right|^{2}+2\left|g_{2}\right|\left|g_{1}\right| \cos \left(\frac{2 \pi}{3}\right) \\
& =\left(\left|g_{2}\right|-\frac{1}{2}\left|g_{1}\right|\right)^{2}+\frac{3}{4}\left|g_{1}\right|^{2} .
\end{aligned}
$$

Thus, the outage probability can be upper bounded

$$
\begin{aligned}
& P_{2}^{\text {out }}(\rho) \leq \operatorname{Pr}\left\{\left(\left|g_{2}\right|-\frac{1}{2}\left|g_{1}\right|\right)^{2}+\frac{3}{4}\left|g_{1}\right|^{2}<\epsilon_{0} \rho^{-1}\right\} \\
& \stackrel{(a)}{<} \operatorname{Pr}\left\{\left(\left|g_{2}\right|-\frac{1}{2}\left|g_{1}\right|\right)^{2}<\epsilon_{0} \rho^{-1}, \frac{3}{4}\left|g_{1}\right|^{2}<\epsilon_{0} \rho^{-1}\right\} \\
& =\operatorname{Pr}\left\{\left|g_{1}\right|^{2}<\omega_{1} \rho^{-1}\right\} \\
& \cdot \operatorname{Pr}\left\{\left(\left|g_{2}\right|-\frac{1}{2}\left|g_{1}\right|\right)^{2}<\epsilon_{0} \rho^{-1}|| g_{1} \mid<\sqrt{\omega_{1}} \rho^{-\frac{1}{2}}\right\},
\end{aligned}
$$

where $\omega_{1} \triangleq \frac{4}{3} \epsilon_{0}$ and $(a)$ follows from $\max (x, y)<x+y$ for $x, y>0$. From (3), as $\rho \rightarrow \infty$, we obtain

$$
\operatorname{Pr}\left\{\left|g_{1}\right|^{2}<\omega_{1} \rho^{-1}\right\} \approx-\omega_{1} \rho^{-1} \ln \left(\omega_{1} \rho\right) \doteq \rho^{-1} .
$$

Furthermore, by setting $g_{n}=g_{2}, a=\frac{1}{2}\left|g_{1}\right|, b=\epsilon_{0}$ and $c=\frac{1}{2} \sqrt{\omega_{1}}$ in Lemma 2. (a), we have

$$
\operatorname{Pr}\left\{\left(\left|g_{2}\right|-\frac{1}{2}\left|g_{1}\right|\right)^{2}<\epsilon_{0} \rho^{-1}|| g_{1} \mid<\sqrt{\omega_{1}} \rho^{-\frac{1}{2}}\right\} \leq \rho^{-1} \text {. }
$$

\footnotetext{
${ }^{3}$ Throughout this letter, each parameter is acquiescently not a function of $\rho$, i.e., its value does not change with $\rho$, unless otherwise stated.
} 
Substituting (8) and (9) into (7), we obtain $P_{2}^{\text {out }}(\rho) \dot{\leq} \rho^{-2}$. Therefore, we evince that $d_{2}(3) \geq 2$. Since the maximum diversity order is 2 when $N=2$, we conclude that $d_{2}(3)=2$.

\section{B. $N \geq 2$}

The result for $N=2$ can be generalized to the case $N \geq 2$, as stated in the following proposition.

Proposition 1: For $\forall \lambda \in \mathbb{R}^{+}$and $\forall N \geq 2$, we have

$$
\operatorname{Pr}\left\{\left|G_{N}\right|^{2}<\lambda \rho^{-1}\right\} \dot{\leq} \rho^{-N} .
$$

Proof 3: This proposition can be proved using mathematical induction. We consider the following induction steps.

1) $N=2$ : This case is considered in Section [III-A and (10) holds by replacing $\epsilon_{0}$ with $\lambda$.

2) $N=k-1$ : When $N=k-1, k \geq 3$, we assume that (10) holds true, i.e.,

$$
\operatorname{Pr}\left\{\left|G_{k-1}\right|^{2}<\lambda \rho^{-1}\right\} \dot{\leq} \rho^{-(k-1)}, \forall \lambda \in \mathbb{R}^{+},
$$

where $G_{k-1}=\sum_{n=1}^{k-1} g_{n}$. This is the induction hypothesis in our problem formulation.

3) $N=k$ : Based on the induction hypothesis corresponding to $N=k-1$, we proceed as follows. Based on Lemma 11, we have $\arg \left(G_{k-1}\right) \in\left[-\frac{\pi}{3}, \frac{\pi}{3}\right]$, and hence $\left|G_{k}\right|^{2}=\left|g_{k}+G_{k-1}\right|^{2}$ can be bounded as follows

$$
\left|G_{k}\right|^{2} \geq\left(\left|g_{k}\right|-\frac{1}{2}\left|G_{k-1}\right|\right)^{2}+\frac{3}{4}\left|G_{k-1}\right|^{2},
$$

with the aid of steps similar to (6). In addition, similar to (7), for $\forall \lambda \in \mathbb{R}^{+}$, we can obtain

$$
\begin{gathered}
\operatorname{Pr}\left\{\left|G_{k}\right|^{2}<\lambda \rho^{-1}\right\} \leq \operatorname{Pr}\left\{\left|G_{k-1}\right|^{2}<\frac{4}{3} \lambda \rho^{-1}\right\} \cdot \operatorname{Pr}\{ \\
\left.\left(\left|g_{k}\right|-\frac{1}{2}\left|G_{k-1}\right|\right)^{2}<\lambda \rho^{-1}|| G_{k-1} \mid<\left(\frac{4}{3} \lambda\right)^{\frac{1}{2}} \rho^{-\frac{1}{2}}\right\} .
\end{gathered}
$$

Finally, by applying Lemma 2-(a) and (11) to (13), we obtain $\operatorname{Pr}\left\{\left|G_{k}\right|^{2}<\lambda \rho^{-1}\right\} \dot{\leq} \rho^{-k}$ for $\forall \lambda \in \mathbb{R}^{+}$. This completes the proof.

Based on Proposition $11 P_{N}^{\text {out }}(\rho) \dot{\leq} \rho^{-N}$, i.e., $d_{N}(3) \geq N$ holds for $\forall N \geq 2$. Since the achievable diversity order cannot exceed $N$, we have the following theorem.

Theorem 1: If the number of quantization levels is $L \geq 3$, the full diversity order of $N$ can be achieved, i.e., $d_{N}(L)=N$, $\forall L \geq 3, \quad N \geq 2$.

Now, one may wonder whether $L=3$ is the minimum required number of phase quantization levels for achieving the full diversity order. To answer this question, we analyze the setup for $L=2$ in the next section.

\section{DIVERSITY ORDER FOR $L=2$}

This section aims to prove that the full diversity of $N$ cannot be achieved if only two quantization levels are used. If $L=2$, in particular, we have $\mathcal{F}=\{0, \pi\}$ and $\Theta_{n} \in\left[-\frac{\pi}{2}, \frac{\pi}{2}\right]$, $\forall n \in\{1, \ldots, N\}$. To prove this result, we first compute a lower bound for the outage probability, and prove that the corresponding exponential order is $-\frac{N+1}{2}$. Based on this result, we conclude that the diversity order cannot exceed $\frac{N+1}{2}$.

\section{A. $N=2$}

As mentioned, we need to identify a lower bound for the outage probability. For fixed $\left|g_{1}\right|$ and $\left|g_{2}\right|,\left|G_{2}\right|$ attains its minimum value if $\left|\Theta_{1}-\Theta_{2}\right|=\pi$. Therefore, we are interested in computing the outage probability conditioned on the event that $\left|\Theta_{1}-\Theta_{2}\right|$ is close to $\pi$. This is provided in the following proposition.

Proposition 2: At high SNR, define the event $\varepsilon_{1}$ as follows

$$
\begin{gathered}
\varepsilon_{1} \triangleq\left\{\Theta_{i} \in\left[-\frac{\pi}{2},-\frac{\pi}{2}+\theta\right], \Theta_{j} \in\left[\frac{\pi}{2}-\theta, \frac{\pi}{2}\right],\right. \\
(i, j)=(1,2) \text { or }(2,1)\},
\end{gathered}
$$

where $\theta \triangleq \rho^{-\frac{1}{2}}$. Conditioned on the event $\varepsilon_{1}$, we have

$$
\operatorname{Pr}\left\{\left|G_{2}\right|^{2}<\epsilon_{0} \rho^{-1} \mid \varepsilon_{1}\right\} \dot{\geq} \rho^{-\frac{1}{2}} .
$$

Proof 4: Without loss of generality for the event $\varepsilon_{1}$, we assume $\Theta_{1} \in\left[-\frac{\pi}{2},-\frac{\pi}{2}+\theta\right], \Theta_{2} \in\left[\frac{\pi}{2}-\theta, \frac{\pi}{2}\right]$. Hence $\pi-2 \theta \leq \Theta_{2}-\Theta_{1} \leq \pi$ and $\left|G_{2}\right|^{2}$ can be upper bounded as follows

$$
\begin{aligned}
& \left|G_{2}\right|^{2}=\left|g_{1}\right|^{2}+\left|g_{2}\right|^{2}+2\left|g_{1}\right|\left|g_{2}\right| \cos \left(\Theta_{2}-\Theta_{1}\right) \\
& \leq\left|g_{1}\right|^{2}+\left|g_{2}\right|^{2}-2 \cos (2 \theta)\left|g_{1}\right|\left|g_{2}\right| \\
& =\left(\left|g_{1}\right|-\cos (2 \theta)\left|g_{2}\right|\right)^{2}+\sin ^{2}(2 \theta)\left|g_{2}\right|^{2} \\
& <\left(\left|g_{1}\right|-\cos (2 \theta)\left|g_{2}\right|\right)^{2}+4 \rho^{-1}\left|g_{2}\right|^{2} \triangleq \Lambda\left(\left|g_{1}\right|^{2},\left|g_{2}\right|^{2}\right),
\end{aligned}
$$

where the last step is based on the definition $\theta=\rho^{-\frac{1}{2}}$ and the fact that $\sin (\theta)<\theta$. Furthermore, we have

$$
\begin{aligned}
& \operatorname{Pr}\left\{\left|G_{2}\right|^{2}<\epsilon_{0} \rho^{-1} \mid \varepsilon_{1}\right\} \\
&> \operatorname{Pr}\left\{\Lambda\left(\left|g_{1}\right|^{2},\left|g_{2}\right|^{2}\right)<\epsilon_{0} \rho^{-1}\right\} \\
& \stackrel{(a)}{>} \operatorname{Pr}\left\{\Lambda\left(\left|g_{1}\right|^{2},\left|g_{2}\right|^{2}\right)<\epsilon_{0} \rho^{-1}, \omega_{2} \leq\left|g_{2}\right|^{2} \leq 2 \omega_{2}\right\} \\
&=\operatorname{Pr}\left\{\omega_{2} \leq\left|g_{2}\right|^{2} \leq 2 \omega_{2}\right\} \cdot \operatorname{Pr}\left\{\Lambda\left(\left|g_{1}\right|^{2},\left|g_{2}\right|^{2}\right)\right. \\
&\left.\qquad \epsilon_{0} \rho^{-1}\left|\sqrt{\omega_{2}} \leq\right| g_{2} \mid \leq \sqrt{2 \omega_{2}}\right\} \\
& \stackrel{(b)}{>}\left[F_{\left|g_{2}\right|^{2}}\left(2 \omega_{2}\right)-F_{\left|g_{2}\right|^{2}}\left(\omega_{2}\right)\right] \cdot \operatorname{Pr}\left\{\left(\left|g_{1}\right|\right.\right. \\
&\left.\left.\quad-\cos (2 \theta)\left|g_{2}\right|\right)^{2}<\frac{\epsilon_{0} \rho^{-1}}{2}\left|\sqrt{\omega_{2}} \leq\right| g_{2} \mid \leq \sqrt{2 \omega_{2}}\right\} \\
& \doteq \rho^{-\frac{1}{2}},
\end{aligned}
$$

where $\omega_{2} \triangleq \frac{\epsilon_{0}}{16} ;(a)$ holds since $\operatorname{Pr}\{A\}>\operatorname{Pr}\{A, B\}$ for all events $A$ and $B$ satisfying $B \nsubseteq A$, and is used to limit the range of $\left|g_{2}\right|$ to $\left[\sqrt{\omega_{2}}, \sqrt{2 \omega_{2}}\right]$ so that $\left|g_{2}\right| \doteq \rho^{0}$ holds for $\forall\left|g_{2}\right| \in\left[\sqrt{\omega_{2}}, \sqrt{2 \omega_{2}}\right]$ and Lemma 2-(b) can be applied in the last step; (b) is due to the fact that $4 \rho^{-1}\left|g_{2}\right|^{2}<\frac{\epsilon_{0} \rho^{-1}}{2}$ if $\left|g_{2}\right|<\sqrt{2 \omega_{2}}$; and the last step is based on Lemma 2 (b) (setting $a=\cos (2 \theta)\left|g_{2}\right|$ and $b=\epsilon_{0} / 2$ ).

Remark 3: The motivation for setting $\theta=\rho^{-\frac{1}{2}}$ is to make sure that the term $4 \theta^{2}\left|g_{2}\right|^{2}$ in (16) satisfies $4 \theta^{2}\left|g_{2}\right|^{2}<\epsilon_{0} \rho^{-1}$ even if $\left|g_{2}\right|^{2} \doteq \rho^{0}$. In this case, $\left|g_{2}\right|^{2}$ can take any values in the range $\left[\omega_{2}, 2 \omega_{2}\right]$, so that $\left|g_{2}\right|^{2} \doteq \rho^{0}$ is satisfied and the conditional outage probability $\operatorname{Pr}\left\{\left|G_{2}\right|^{2}<\epsilon_{0} \rho^{-1} \mid \varepsilon_{1}\right\}$ can be exponentially lower bounded by $\rho^{-\frac{1}{2}}$ as stated in (17) . 
Moreover, the probability of the event $\varepsilon_{1}$ can be computed as $\operatorname{Pr}\left\{\varepsilon_{1}\right\}=2\left(\frac{\theta}{\pi}\right)^{2} \doteq \rho^{-1}$, since

$$
\operatorname{Pr}\left\{\Theta_{i} \in\left[-\frac{\pi}{2},-\frac{\pi}{2}+\theta\right]\right\}=\operatorname{Pr}\left\{\Theta_{i} \in\left[\frac{\pi}{2}, \frac{\pi}{2}-\theta\right]\right\}=\frac{\theta}{\pi},
$$

where $i=1,2$. Therefore, based on Proposition 2 the outage probability can be exponentially lower bounded as follows

$$
P_{2}^{\text {out }}(\rho) \geq \operatorname{Pr}\left\{\varepsilon_{1}\right\} \cdot \operatorname{Pr}\left\{\left|G_{2}\right|^{2}<\epsilon_{0} \rho^{-1} \mid \varepsilon_{1}\right\} \geq \rho^{-\frac{3}{2}} .
$$

Thus, $d_{N}(2) \leq \frac{3}{2}$, which is smaller than the theoretical full diversity order of $N=2$.

\section{B. $\quad N \geq 2$}

Proposition 2 can be generalized to the case $N \geq 2$, as shown in the following corollary.

Corollary 1: At high SNR, define the event $\varepsilon_{2}$ as follows

$$
\begin{array}{r}
\varepsilon_{2} \triangleq\left\{\Theta_{i} \in\left[-\frac{\pi}{2},-\frac{\pi}{2}+\theta\right],\left\{\Theta_{j}\right\}_{j \in\{1, \ldots, N\}, j \neq i} \in\right. \\
\left.\left[\frac{\pi}{2}-\theta, \frac{\pi}{2}\right], \forall i \in\{1, \ldots, N\}\right\} .
\end{array}
$$

Conditioned on the event $\varepsilon_{2}$, we have $\operatorname{Pr}\left\{\left|G_{N}\right|^{2}<\epsilon_{0} \rho^{-1} \mid \varepsilon_{2}\right\} \geq \rho^{-\frac{1}{2}}$.

Proof 5: For the event $\varepsilon_{2}$, assume $\Theta_{1} \in\left[-\frac{\pi}{2},-\frac{\pi}{2}+\theta\right]$ and $\left\{\Theta_{j}\right\}_{j \in\{2, \ldots, N\}} \in\left[\frac{\pi}{2}-\theta, \frac{\pi}{2}\right]$ without loss of generality. Moreover, define $\tilde{G}_{N-1} \triangleq \sum_{j=2}^{N^{2}} g_{j}$, and hence $G_{N}=g_{1}+$ $\tilde{G}_{N-1}$. According to Lemma 1 we obtain $\arg \left(\tilde{G}_{N-1}\right) \in\left[\frac{\pi}{2}-\right.$ $\left.\theta, \frac{\pi}{2}\right]$. Therefore, by using a similar analytical derivation as for Proposition 2 the corollary can be proved by replacing $g_{2}$ with $\tilde{G}_{N-1}$ in (16) and 17).

In addition, from (18), the probability of the event $\varepsilon_{2}$ is $\operatorname{Pr}\left\{\varepsilon_{2}\right\}=N\left(\frac{\theta}{\pi}\right)^{N} \doteq \rho^{-\frac{N}{2}}$. Therefore, we obtain

$$
P_{N}^{\text {out }}(\rho) \geq \operatorname{Pr}\left\{\varepsilon_{2}\right\} \cdot \operatorname{Pr}\left\{\left|G_{N}\right|^{2}<\epsilon_{0} \rho^{-1} \mid \varepsilon_{2}\right\} \geq \rho^{-\frac{N+1}{2}} .
$$

From 21, the upper bound of the diversity order for $L=2$ can be summarized in the following theorem.

Theorem 2: If the number of quantization levels is $L=2$, the diversity order is upper bounded by $d_{N}(2) \leq \frac{N+1}{2}, \forall N \geq$ 2 .

Remark 4: Combing Theorem 1 and Theorem 2, the minimum required number of phase quantization levels to achieve the full diversity order of $N$ is equal to $L=3$.

\section{IMPACT OF DIRECT LINK}

If the direct link between $S$ and $D$ cannot be ignored, Theorem 1 and Theorem 2 can be modified as follows.

Let $h_{S D}$ denote the channel of the direct link. The optimal value of $\phi_{n}$ in Section 【I is $\phi_{n}^{*}=\arg \left(h_{S D}\right)-$ $\arg \left(\left[\mathbf{h}_{S I}\right]_{n}\left[\mathbf{h}_{I D}\right]_{n}\right), n \in\{1, \ldots, N\}$. Moreover, the SNR is $\gamma_{D}=\rho|| h_{S D}\left|+\eta \sqrt{\Omega_{S} \Omega_{I}} G_{N}\right|^{2}$. Based on Lemma 1 $\left|\arg \left(G_{N}\right)\right|$ cannot exceed $\frac{\pi}{2}$ even if $L=2$, so $\gamma_{D} \geq$ $\rho\left(\left|h_{S D}\right|^{2}+\eta^{2} \Omega_{S} \Omega_{I}\left|G_{N}\right|^{2}\right)$ for $\forall L \geq 2$. Based on this lower bound on $\gamma_{D}$, Theorem 1 still holds by replacing the diversity order $N$ with $N+1$.

Furthermore, $\gamma_{D}$ can be upper bounded as $\gamma_{D} \leq \rho\left(\left|h_{S D}\right|+\right.$ $\left.\eta \sqrt{\Omega_{S} \Omega_{I}}\left|G_{N}\right|\right)^{2}$. Therefore, Theorem 2 holds by replacing the upper bound $\frac{N+1}{2}$ with $\frac{N+3}{2}$. The details are omitted due to space limitations.

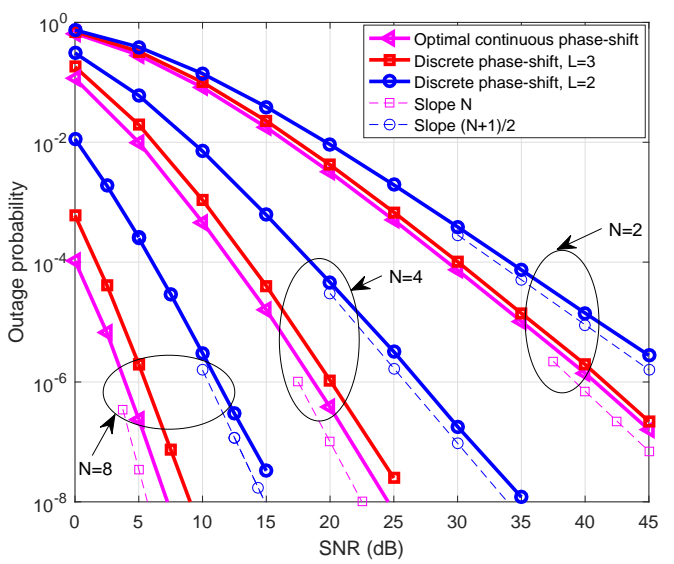

Fig. 1. The outage probability versus the SNR, for different values of $L$ and $N$, where $R_{0}=1$ bpcu.

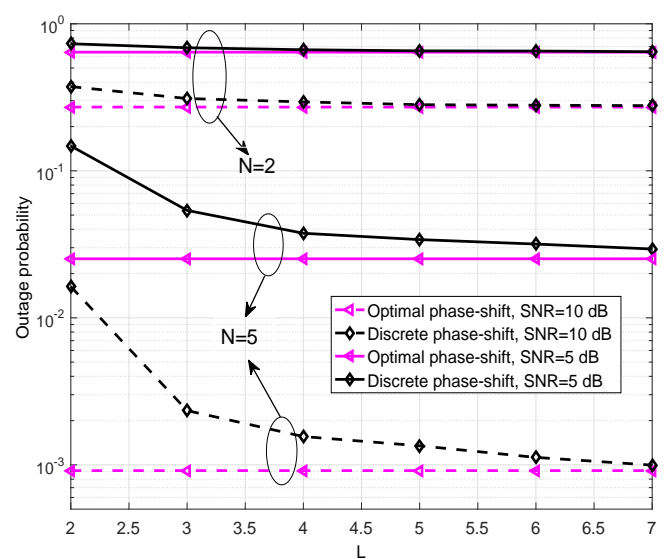

Fig. 2. The outage probability versus $L$, for different values of the transmit SNR and $N$, where $R_{0}=2 \mathrm{bpcu}$.

\section{NUMERICAL RESULTS}

In this section, Monte Carlo simulations are provided to verify the accuracy of the analytical results about the diversity order. The direct link between $S$ and $D$ does not exist, and we set $\left[\mathbf{h}_{S I}\right]_{n} \sim \mathcal{C N}(0,1)$ and $\left[\mathbf{h}_{I D}\right]_{n} \sim \mathcal{C N}(0,0.5), \forall n \in$ $\{1, \ldots, N\}$, i.e., $\Omega_{S}=1$ and $\Omega_{I}=0.5$. We also set $\eta=0.8$.

The effect of phase quantization on the diversity order of RIS-aided transmission is illustrated in Fig. 1. For ease of interpretation, we reports the lines with slopes $\frac{N+1}{2}$ and $N$ as well. When $L=2$, for $N=2, N=4$ and $N=8$, we can observe that the slope of the outage probability curves is not smaller than $-1.5,-2.5$ and -4.5 (i.e., the diversity order does not exceed 1.5, 2.5 and 4.5) at high SNRs, respectively. This observation is consistent with Theorem 2. The main reason is that the phase error is uniformly distributed over $\left[-\frac{\pi}{2}, \frac{\pi}{2}\right]$, and there exists a probability that each phase error is close to the boundary of $-\frac{\pi}{2}$ or $\frac{\pi}{2}$. In this case, the amplitudes of the channel coefficients mutually cancel out, which results in a loss of the diversity order. When $L \geq 3$, on the other hand, we observe that the corresponding curves are parallel to the curves obtained in the absence of phase errors. We conclude that the full diversity order can be achieved as long as $L \geq 3$, which is consistent with Theorem 1 
In Fig. 2 the outage probability as a function of the phase quantization level $L$ is shown. We note that the outage performance is a decreasing function of $L$. As $L$ increases, the outage probability approaches the lower bound with perfect phase shifts. For both setups $N=2$ and $N=5$, the outage probability has the largest values if $L=2$. When $N=2$, the outage probability approaches the lower bound with perfect phase shifts provided that $L \geq 3$. When $N=5$, the gap between the curve with phase errors and the curve with perfect phase shifts is larger. However, the gap reduces as $L$ increases. Therefore, the quantization errors for phase shifts of the RIS do not lead to significant outage performance loss, which is a promising finding for the deployment of RISs in wireless systems.

\section{CONCLUSION}

This letter investigated the diversity order of RIS-aided communication systems with discrete phase shifts. The main contribution of this letter was to unveil the minimum number of phase quantization levels to achieve full diversity. In particular, the full diversity order is proved to be achievable, if and only if at least three quantization levels are used. Simulation results verified the theoretical finding and showed that the outage performance loss is negligible even for moderate values of the quantization levels.

\section{APPENDIX A}

\section{PROOF OF LEMMA 2}

We consider different values of $a$ as follows.

1) For $0<a<c \rho^{-\frac{1}{2}}$ : We have

$$
\begin{aligned}
& \operatorname{Pr}\left\{\left(\left|g_{n}\right|-a\right)^{2}<b \rho^{-1}\right\} \\
= & \operatorname{Pr}\left\{-\sqrt{b} \rho^{-\frac{1}{2}}+a<\left|g_{n}\right|<\sqrt{b} \rho^{-\frac{1}{2}}+a\right\} \\
\leq & \operatorname{Pr}\left\{\left|g_{n}\right|^{2}<\left(\sqrt{b} \rho^{-\frac{1}{2}}+c \rho^{-\frac{1}{2}}\right)^{2}\right\} \\
= & 1-2(\sqrt{b}+c) \rho^{-\frac{1}{2}} K_{1}\left(2(\sqrt{b}+c) \rho^{-\frac{1}{2}}\right) \\
\approx & -(\sqrt{b}+c)^{2} \rho^{-1} \ln \left((\sqrt{b}+c)^{2} \rho^{-1}\right),
\end{aligned}
$$

as $\rho \rightarrow \infty$, where the last step is based on (3). Thus, $\operatorname{Pr}\left\{\left(\left|g_{n}\right|-a\right)^{2}<b \rho^{-1}\right\} \leq \rho^{-1}$.

2) For $a>0$ and $a \doteq \rho^{0}$ : Based on 22, we have

$$
\begin{aligned}
& \operatorname{Pr}\left\{\left(\left|g_{n}\right|-a\right)^{2}<b \rho^{-1}\right\} \\
= & \operatorname{Pr}\left\{\left(-\sqrt{b} \rho^{-\frac{1}{2}}+a\right)^{2}<\left|g_{n}\right|^{2}<\left(\sqrt{b} \rho^{-\frac{1}{2}}+a\right)^{2}\right\} \\
= & \tilde{K}_{1}(2 a-z)-\tilde{K}_{1}(2 a+z),
\end{aligned}
$$

where $\tilde{K}_{1}(x) \triangleq x K_{1}(x), z \triangleq 2 \sqrt{b} \rho^{-\frac{1}{2}}$ and $2 a \gg z$ holds as $\rho \rightarrow \infty$. From [17, Eq. 8.446], the function $\tilde{K}_{1}(x)$ has the following series representation:

$$
\tilde{K}_{1}(x)=1-\sum_{k=0}^{\infty} \frac{\left(\frac{x}{2}\right)^{2 k+2}\left(A(k)-\ln \frac{x}{2}\right)}{k !(k+1) !},
$$

where $A(k) \triangleq \frac{1}{2} \psi(k+1)+\frac{1}{2} \psi(k+2)$, and the function $\psi(\cdot)$ is defined in [17, Eq. 8.365.3]. Therefore, by using the Taylor expansion at the point $2 a$, we have

$$
\tilde{K}_{1}(2 a-z)=\tilde{K}_{1}(2 a)-\tilde{K}_{1}^{\prime}(2 a) z+o(z),
$$

where $o(\cdot)$ denotes higher order terms and $\tilde{K}_{1}^{\prime}(2 a)$ can be expressed as follows

$$
\tilde{K}_{1}^{\prime}(2 a)=-\sum_{k=0}^{\infty} \frac{a^{2 k+1}\left[(k+1)(A(k)-\ln a)+\frac{1}{2}\right]}{k !(k+1) !} .
$$

Similarly, $\tilde{K}_{1}(2 a+z)$ can be expressed as

$$
\tilde{K}_{1}(2 a+z)=\tilde{K}_{1}(2 a)+\tilde{K}_{1}^{\prime}(2 a) z+o(z) .
$$

Inserting (26) and (28) into (24), we have

$$
\operatorname{Pr}\left\{\left(\left|g_{n}\right|-a\right)^{2}<b \rho^{-1}\right\} \approx-4 \sqrt{b} \rho^{-\frac{1}{2}} \tilde{K}_{1}^{\prime}(2 a) \doteq \rho^{-\frac{1}{2}} .
$$

\section{REFERENCES}

[1] M. Di Renzo, et al., EURASIP Journal on Wireless Communications and Networking, vol. 2019, no. 1, pp. 1-20, May 2019.

[2] — "Smart radio environments empowered by reconfigurable intelligent surfaces: How it works, state of research, and road ahead," arXiv:2004.09352.

[3] C. Huang, S. Hu, et al., "Holographic MIMO surfaces for 6G wireless networks: opportunities, challenges, and trends," arXiv:1911.12296.

[4] Q. Wu and R. Zhang, "Towards smart and reconfigurable environment: Intelligent reflecting surface aided wireless network," IEEE Commun. Mag., vol. 58, no. 1, pp. 106-112, Nov. 2020.

[5] M. Di Renzo, et al., "Reconfigurable intelligent surfaces vs. relaying: Differences, similarities, and performance comparison," IEEE Open J. Commun. Society, vol. 1, pp. 798-807, June 2020.

[6] Q. Wu and R. Zhang, "Intelligent reflecting surface enhanced wireless network via joint active and passive beamforming," IEEE Trans. Wireless Commun., vol. 18, no. 11, pp. 5394-5409, Nov. 2019.

[7] _ - "Beamforming optimization for wireless network aided by intelligent reflecting surface with discrete phase shifts," IEEE Trans. Communications, vol. 68, no. 3, pp. 1838-1851, Mar. 2020.

[8] Z. Ding, R. Schober, and H. V. Poor, "On the impact of phase shifting designs on IRS-NOMA," IEEE Wireless Commun. Lett. (Early Access).

[9] B. Zheng, Q. Wu, and R. Zhang, "Intelligent reflecting surface-assisted multiple access with user pairing: NOMA or OMA?" IEEE Commun. Lett., vol. 24, no. 4, pp. 753-757, Jan. 2020

[10] Z. Chu, W. Hao, P. Xiao, and J. Shi, "Intelligent reflecting surface aided multi-antenna secure transmission," IEEE Wireless Commun. Lett., vol. 9, no. 1, pp. 108-112, Sep. 2020.

[11] Z. Abdullah, G. Chen, S. Lambotharan, and J. A. Chambers, "A hybrid relay and intelligent reflecting surface network and its ergodic performance analysis," IEEE Wireless Commun. Lett. (Early Access).

[12] M. Badiu and J. P. Coon, "Communication through a large reflecting surface with phase errors," IEEE Wireless Commun. Lett., vol. 9, no. 2, pp. 184-188, Oct. 2020.

[13] H. Zhang, B. Di, L. Song, and Z. Han, "Reconfigurable intelligent surfaces assisted communications with limited phase shifts: How many phase shifts are enough?" IEEE Trans. Veh. Technol., vol. 69, no. 4, pp. 4498-4502, Apri. 2020.

[14] D. Li, "Ergodic capacity of intelligent reflecting surface-assisted communication systems with phase errors," IEEE Commun. Lett. (Early Access).

[15] X. Qian, M. Di Renzo, J. Liu, A. Kammoun, and M. S. Alouini, "Beamforming through reconfigurable intelligent surfaces in single-user MIMO systems: SNR distribution and scaling laws in the presence of channel fading and phase noise," arXiv:2005.07472.

[16] L. Zheng and D. N. C. Tse, "Diversity and multiplexing : A fundamental tradeoff in multiple antenna channels," IEEE Trans. Inform. Theory, vol. 49, pp. 1073-1096, May 2003.

[17] I. S. Gradshteyn and I. M. Ryzhik, Table of Integrals, Series and Products, 6th ed. New York, NY, USA: Academic, 2000. 